\title{
Basic-level categorization and part-whole perception in children
}

\author{
RUTH KIMCHI \\ University of Haifa, Haifa, Israel
}

\begin{abstract}
Tversky \& Hemenway's (1984) claim that parts are central to basic-level categorization suggests that parts should play an important role in the formation of basic-level categories by young children. There is, however, a prevailing claim that young children do not attend to parts, but treat objects as integral wholes. The present study attempted to compare children's ability to categorize at the basic level and their sensitivity to parts and part-whole relations. Nursery school children, kindergarteners, first- and second-graders performed three tasks: (1) sorting objects into basic-level categories, (2) sorting parts that belong to the same object, and (3) matching parts with basic-level objects. Children's performance on parts sorting and part-object matching lagged behind their objects sorting, suggesting that attention to parts per se does not seem to be essential for children's basic-level categorization. Also, by 5 years of age, children showed sensitivity to parts and to part-whole relations, and this sensitivity improved with age.
\end{abstract}

In taxonomies of common objects and living things, categories at one level of abstraction are psychologically better or more useful than more general and more specific categories (Rosch, 1978; Rosch, Mervis, Gray, Johnson, \& Boyes-Braem, 1976). This is the level of "chair" and "dog," as opposed to "furniture" and "animals" or "kitchen chair" and "poodle." There is by now a great deal of evidence attesting to the special status of basiclevel categories, based on perceptual, behavioral, and linguistic measures (e.g., Murphy \& Smith, 1982; Rosch, 1978; Rosch et al., 1976).

Rosch (1978; Rosch et al., 1976) suggested that what sets the basic level apart is that at this level, the two different goals of categorization, maximizing similarity within categories and minimizing similarity between categories, are best achieved. At the basic level, there are many attributes common to all members of the category, but few attributes, if any at all, common to members of other categories. On the other hand, members of superordinate categories share few attributes among each other, and members of subordinate categories share many attributes with members of other categories.

Tversky \& Hemenway (1984) suggested more specifically that what grants special status to the basic-level objects is that objects at this level share many parts with other members of their category, but few with members of other categories.

Children tend to acquire basic-level categories prior to superordinate- and subordinate-level categories (e.g.,

I would like to thank David Freund for his assistance in executing the experiment reported here, the children and the teachers of Kibbutz Kefar Glickson for their participation and cooperation, and Natan Chen for the line drawings of the parts. Requests for reprints should be addressed to Ruth Kimchi, Department of Psychology, University of Haifa, Haifa 31905, Israel.
Mervis \& Crisafi, 1982). Most of children's first learned words are basic-level terms (Clark, 1973; Rosch et al., 1976), and children as young as 3 years of age are able to categorize at the basic level, whereas they have a great difficulty categorizing at the superordinate or subordinate levels (Rosch et al., 1976).

If young children are able to categorize at the basic level (e.g., Rosch et al., 1976), and parts are central to the determination of basic-level categorization (Tversky \& Hemenway, 1984), then young children should be able to attend to parts (see also Mervis \& Greco, 1984). It is even logically possible that children apprehend parts before being able to categorize at the basic level. This, however, seems to be contradictory to the claim that there is a developmental trend from a state of global, holistic perception to a state of increased differentiation (e.g., Gibson, 1969; Werner, 1957). This claim has been supported by findings demonstrating that young children, even at the age of 6 years, tend to classify stimuli holistically on the basis of overall similarity relations, whereas older children and adults classify dimensionally (e.g., Kemler Nelson, 1989; Smith, 1983). The stimuli used in these studies varied along dimensions (e.g., color and size), and the results suggest that young children do not attend to dimensions.

However, dimensions and parts are not identical (see Garner, 1978, and Treisman, 1986, for eloquent discussions of these different aspects of a stimulus). It is possible that young children are not sensitive to dimensions, but they are sensitive to parts. For example, Ward (1989) found children to behave analytically in concept learning tasks when parts of objects (e.g., wings, eyes, noses, etc.), rather than dimensions, were varied.

The question of whether children focus on "parts" or "wholes" has been also studied by using artificial stimuli in which larger figures are constructed by the suitable arrangement of smaller figures (e.g., a "person" constructed 
from fruit "parts," or a rectangle constructed from squares). The results of these studies show that young children sometimes focus on wholes (e.g., Lowe, 1973) and sometimes focus on parts (e.g., Elkind, Koegler, \& Go, 1964; Prather \& Bacon, 1986; Vurpillot, 1976). However, many of these studies have been criticized for lacking proper control over stimulus material and for relying on verbal skills (see Prather \& Bacon, 1986; Vurpillot, 1976). Furthermore, the constituents of many of the stimuli that were used do not necessarily function perceptually as parts of the overall figure (see Kimchi, 1990).

The present study attempted to examine children's apprehension of parts and part-whole relations of basic-level objects and to compare it with their ability to categorize at the basic level. Children at four levels of age performed an objects sorting task, a parts sorting task, and a part-object matching task. Because a ceiling in correct sorting of basiclevel objects was reached by the age of 4 when an oddity task was used (Rosch et al., 1976), a somewhat more difficult task was used in order to get sorting differences among the younger children to allow computations of correlations between performance on the different tasks. The objects used were natural and are well-known to young children.

\section{METHOD}

\section{Subjects}

A total of 40 children participated in the experiment: 11 nursery school children ( 6 males and 5 females; mean age, 3 years 2 months; range, 2 years 6 months to 3 years 10 months), 10 kindergarteners (4 males and 6 females; mean age, 5 years 5 months; range, 4 years 8 months to 6 years 1 month), 10 first-graders ( 4 males and 6 females; mean age, 7 years; range, 6 years 11 months to 7 years 5 months), and 9 secondgraders ( 3 males and 6 females; mean age, 8 years; range, 7 years 7 months to 8 years 4 months). All children attended a school in a kibbutz.

\section{Stimuli and Tasks}

The stimuli for the objects sorting were color photographs of objects, which were photographed in a canonical perspective. Six basic-level categories were used (see Table 1). There were five pictures for each basiclevel category. Twelve sets of five pictures each were constructed. Three pictures in a set could be correctly grouped at one basic-level category (e.g., three birds), and two pictures could be correctly grouped at another basic-level category (e.g., two cars). The sets were constructed in such a way that each category was combined with any other category, with the restriction that each category appeared four times, and each of the five pictures available per basic-level category appeared twice. Pictures from the same basic-level category in a set were not of the same color.

For each object, three parts were chosen (see Table 1) on the basis of the goodness ratings obtained by Tversky and Hemenway (1984) and according to visibility. For example, although Tversky and Hemenway's subjects rated "engine"' high in goodness as a part of a car, it was not used in the present experiment because it is an internal part and therefore not visible from an external view of a car. For the part-object match-

Table 1

The Basic-Level Objects and Their Parts Used in the Experiment

\begin{tabular}{llll}
\hline Object & \multicolumn{3}{c}{ Parts } \\
\hline Bird & Beak & Wings & Legs \\
Dog & Tail & Legs & Nose \\
Car & Tire & Wheels & Headlight \\
Kettle & Spout & Handle & Cover \\
Flower & Petals & Stem & Leaves \\
Chair & Seat & Back & Legs \\
\hline
\end{tabular}

ing, black-ink line drawings of two parts per object (the first two parts listed for each object in Table 1), 12 drawings altogether, were used. Each drawing was presented along with a set of five pictures, each picture from a different basic-level category, chosen randomly from the available five pictures per basic-level category, with the restriction that pictures in a set were not of the same color. The children were required to match the part drawing with the appropriate object. The stimuli for the parts sorting were close-up photographs of parts, three parts per object (see Table 1). Two pictures in a triad could be correctly grouped as parts of the same object (e.g., a beak and a wing), and the third picture was a part of another basic-level object (e.g., a tire). Twelve sets of triads were constructed so that parts of each object were combined with a part of any other object, with the restriction that each part appeared twice across the 12 sets. Each picture (or drawing) was mounted on a $17 \times 12 \mathrm{~cm}$ white card.

\section{Procedure}

The subjects were tested individually on each task. All of the subjects performed objects sorting first, then part-object matching, and finally, parts sorting. The experimenter sat opposite the child at a small table. In objects sorting, the child was presented with sets of five pictures, one set at a time, and was told to put together the objects that "belong together, that are alike, that are the same kind of thing." The experimenter also told the child to try and find a place for all of the objects. On each trial of the part-object matching, the experimenter placed a part drawing on the table in front of the child and said "See this?' He then placed five pictures of objects in a row below the drawing. The experimenter pointed again to the part and told the child to put it "where it belongs" (pointing to the five objects). In parts sorting, the child was presented with triads, one triad at a time, and was told to put together "the two that belong together, that belong to the same kind of thing."

The sets of pictures for each task were presented in a different random order to each subject, and the order of pictures within sets was shuffled between subjects. Each task was preceded by two practice trials to ensure that the subject understood the task to be performed.

\section{RESULTS}

The mean percent correct responses for each task for the four age levels are presented in Table 2. A strict criterion was used in evaluating the children's performance on the objects sorting: Only a sorting of all five objects in a set into two complete groups was considered a correct response. There were no sex differences, therefore data for both sexes were combined in the analyses. A twoway analysis of variance (ANOVA) mixed design, which treated age as a between-subject factor and task as a within-subject factor, yielded a significant effect of age $[F(3,36)=14.57, p<.0001]$, a significant effect of task $[F(2,72)=23.68, p<.0001]$, and a significant interaction between task and age $[F(6,72)=2.37, p<.04]$.

A breakdown of the interaction between age and task revealed that performance on each task improved with age $[F(3,36)=5.88, p<.008$, for objects sorting; $F(3,36)$ $=28.37, p<.0001$, for part-object matching; $F(3,36)$ $=12.48, p<.0001$, for parts sorting]. However, pairwise comparisons using the Duncan procedure showed different trends for the different tasks. The nursery school children's performance on objects sorting was inferior to that of the three older age groups, and no significant difference was detected between the performance of the three older age groups. On the other hand, the performance of the first- and second-graders on part-object matching and on parts sorting was superior to that of the kindergar- 
Table 2

Mean Percent Correct Responses in Each Task for Each Age Group

\begin{tabular}{lccc}
\hline & \multicolumn{3}{c}{ Task } \\
\cline { 2 - 4 } \multicolumn{1}{c}{ Age Group } & Objects & $\begin{array}{c}\text { Part-Object } \\
\text { Matching }\end{array}$ & $\begin{array}{c}\text { Parts } \\
\text { Sorting }\end{array}$ \\
\hline Nursery School & 65 & 42 & 52 \\
Kindergarten & 94 & 75 & 68 \\
First Grade & 95 & 85 & 80 \\
Second Grade & 96 & 89 & 85 \\
\hline
\end{tabular}

teners, and kindergarteners performed significantly better than nursery school children.

There was a significant difference between performance on the three tasks for the nursery school children $[F(2,20)$ $=6.98, p<.005]$, the kindergarteners $[F(2,18)=$ $25.07, p<.0001]$, and the first-graders $[F(2,18)=$ $6.63, p<.007]$. Post hoc comparisons using the Duncan procedure indicated that nursery school children and kindergarteners performed objects sorting significantly better than they did the two other tasks. The first-graders' performance on objects sorting was superior to their performance on parts sorting, but it did not differ significantly from their performance on part-object matching. No significant difference between performance on the three tasks was found for the second-graders $[F(2,16)=$ $2.08, p>.15]$.

The first- and second-graders' performance on objects sorting reached ceiling: Nine of the 10 first-graders, and 8 of the 9 second-graders were $100 \%$ correct. Somewhat more variability was present in the kindergarten group: Five kindergarteners were $100 \%$ correct, and 5 were $88 \%$ correct. The greatest variability was present in the nursery school group: Only 3 of the 11 children were $100 \%$ correct, 4 performed below chance level, and 4 performed above chance level.

Pearson correlations between performance on the tasks were computed for the two younger groups. For the nursery school children, the results indicated a significant correlation between object sorting and parts sorting $(r=.75$, $p<.009)$, between object sorting and part-object matching $(r=.73, p<.015)$, and between parts sorting and part-object matching $(r=.87, p<.0004)$. The correlations computed for the kindergarteners were very low, and none reached significance.

When a more lenient criterion was used for evaluating performance on object sorting so that sorting the objects into at least one complete group was considered a correct response, the nursery school children's level of performance increased from $65 \%$ to $82 \%$ (5 of the 11 children were $100 \%$ correct, 3 were $88 \%$ correct, 1 was $63 \%$ correct, and only 1 performed below chance level), and that of the kindergarteners increased from $94 \%$ to $95 \%$ (6 of the 10 children were $100 \%$ correct, and 4 were $88 \%$ correct). The performance of the first- and second-graders remained the same. A two-way ANOVA (task $\times$ age) yielded similar results to the ones reported above: a significant effect of age $[F(3,36)=13.40, p<.0001]$, a significant effect of task $[F(2,72)=46.28, p<.0001]$, and a significant interaction between age and task $[F(6,72)$ $=5.33, p<.0001]$. Both a breakdown of the interaction and Duncan post hoc comparisons also revealed the same pattern of results, except that there was no significant effect of age on performance of objects sorting.

The correlations between performance on object sorting, using the lenient criterion, and the two other tasks were positive $(r=.68, p<.025$, for the correlation between objects sorting and part-object matching, and $r=.56, p<.071$, for the correlation between objects sorting and parts sorting).

\section{DISCUSSION}

The present findings have implications for two issues: the role of parts in children's basic-level categorization and children's sensitivity to partonomic structure. Regarding the implications for the first issue, Tversky and Hemenway's (1984) claim that parts are central for basic-level categorization implies that if young children are able to categorize at the basic level, then they should be able to attend to parts. It is even logically possible that they apprehend parts before they are able to categorize at the basic level. The present results seem to rule out this logical possibility. The 3-year-old children sorted basic-level objects correctly a mean of $65 \%$, which is significantly better than chance $(p<.001)$; by a more lenient criterion, they were correct a mean of $82 \%$. The same children performed at chance level on parts sorting and on part-object matching. Clearly, by the age that children's performance on basic-level categorization was almost perfect, they still had difficulties with the sorting of parts and with identifying part-whole relations. Therefore, it is unlikely that apprehension of parts and part-whole relations precedes children's ability to categorize at the basic level.

The present results, however, do not rule out the possibility that parts may have some role in children's basic-level categorization. When individual differences were found in children's basic-level categorization (i.e., for the nursery school group), the results indicated that children who were better at objects sorting were also better at parts sorting and at matching parts to objects. In addition, by the age of 5 , at which children's objects sorting was very high, performance on parts sorting and part-object matching was significantly better than chance. Tversky (1989) also presented a finding suggesting that parts may play a role in children's categorization. She found that 5-year-old children were better able to form superordinate categories when category members shared parts than when category members did not share parts. However, both the present finding and that of Tversky are subject to alternative explanations. The high correlations found in the present experiment for the 3 -year-old children may be due to individual differences in the young children's general ability to understand and execute the tasks at hand, rather than in their attention to parts. Tversky's (1989) finding is difficult to interpret because having parts in common is correlated with other characteristics such as overall shape and function (see also Markman, 1989; Tversky, 1989; Tversky \& Hemenway, 1984). Perhaps it is the overall shape or the function that dominated children's attention, rather than the parts per se. This seems to be an inherent difficulty in assessing the role of parts in the categorization of natural objects.

Taken together, the present results suggest that parts alone cannot account for children's categorization performance. Possibly, the basic level has a special psychological status, for children as well as for adults, because several criteria tend to converge at this level (see also Markman, 1989; Rosch et al., 1976).

With regard to the implications for evaluating children's sensitivity to partonomic structure, although mean percentage of correct responses on parts sorting and part-object matching of the 3-year-old children was at chance level, the 5-year-old children performed significantly better than chance (a mean of $68 \%, p<.001$, and a mean of $75 \%, p<.001$, for parts sorting and part-object matching, respectively), and early school-age children already performed satisfactorily. These findings suggest that children, at least by the age of 5 , are sensitive to parts and to part-whole relations. These results are generally similar to those obtained by Tversky (1989), who used different tasks. Tversky asked chil- 
dren to detect missing parts in drawings of common objects (Experiments 3 and 4). Her preschool children (4 and 6 years old) failed to detect missing parts, whereas her early school-age children ( 7 and 8 years old) performed quite well. The only discrepancy between the present results and those of Tversky is that the present 5-year-old children already showed sensitivity to parts, whereas Tversky's 6-year-old children performed at chance level. This discrepancy may be due to the differences in the tasks used and, even more likely, to the fact that many of the missing parts in Tversky's experiments were small but functionally significant, whereas in the present experiment, the parts used were perceptually salient. Indeed, young children detected perceptually salient parts faster than they did less salient parts (Tversky, 1989, Experiment 2). Also, when Tversky's young children failed to produce the missing parts, they sometimes provided a large part of the typical setting. Furthermore, when she asked children to produce parts of objects cued by the object's picture (Tversky, 1989, Experiment 5), the parts produced by 4-year-old children tended to be relatively large or distinct.

The present findings suggest that 3-year-old children have difficulty in attending to parts and to part-whole relations. Yet it is possible that the present tasks underestimated children's sensitivity to parts and partwhole relations. Children usually encounter parts of objects in the context of whole objects. The parts sorting task, on the other hand, required them to deal with isolated parts. Young children may be sensitive to part configuration in the context of the whole object but may have much more difficulty in treating isolated parts. The findings of Kimchi (1990), demonstrating that children's perceived organization of hierarchical patterns is similar to that of adults, and the finding of young children's sensitivity to context, demonstrated in their tendency to produce thematic categories (e.g., Markman, 1989), seem to support this hypothesis. The difficulty of the youngest children with the part-object matching task may be due to the fact that they had to deal with line drawings of parts, which seem to involve a certain abstraction of parts. It is possible then that partonomic structure is available to very young children. Whether they can abstract parts and produce partonomic structure is quite another matter.

To conclude, the results of the present experiment show that children as young as $\mathbf{5}$ years of age are sensitive to parts and to part-whole relations, and this sensitivity improves with age. At the same time, the present results seem to suggest that attention to parts per se does not seem to be essential for children's basic-level categorization.

\section{REFERENCES}

Clark, E. V. (1973). What's in a word? On the child's acquisition of semantics in his first language. In T. E. Moore (Ed.), Cognitive development and the acquisition of language. New York: Academic Press.

Elkind, D., Koegler, R. R., \& Go, E. (1964). Studies in perceptual development: II. Part-whole perception. Child Development, 35, 81-90.
Garner, W. R. (1978). Aspects of a stimulus: Features, dimensions, and configurations. In E. Rosch \& B. B. Lloyd (Eds.), Cognition and categorization (pp. 27-48). Hillsdale, NJ: Erlbaum.

GiBson, E. J. (1969). Principles of perceptual learning and development. New York: Appleton-Century-Crofts.

Kemler Nelson, D. G. (1989). The nature and occurrence of holistic processing. In B. Shepp \& S. Ballesteros (Eds.), Object perception: Structure \& process (pp. 357-386). Hillsdale, NJ: Erlbaum.

KIмCHI, R. (1990). Children's perceptual organization of hierarchical visual patterns. European Journal of Cognitive Psychology, 2, 133-149.

LowE, R. C. (1973). A developmental study of part-whole relations in visual perception. Journal of Genetic Psychology, 123, 231-240.

Markman, E. M. (1989). Categorization and naming in children: Problems of induction. Cambridge, MA: MIT Press.

Mervis, B., \& Crisafi, M. A. (1982). Order of acquisition of subordinate, basic, and superordinate level categories. Child Development, 53, 258-266.

Mervis, B., \& Greco, C. (1984). Parts and early conceptual development: Comment on Tversky and Hemenway. Journal of Experimental Psychology: General, 113, 194-197.

MURPHY, G. L., \& SMITH, E. E. (1982). Basic-level superiority in picture categorization. Journal of Verbal Learning \& Verbal Behavior, 21, 1-20.

Prather, P. A., \& Bacon, J. (1986). Developmental differences in part/whole identification. Child Development, 57, 549-558.

Rosch, E. (1978). Principles of categorization. In E. Rosch \& B. B. Lloyd (Eds.), Cognition and categorization (pp. 27-48). Hillsdale, NJ: Erlbaum.

Rosch, E., Mervis, C. B., Gray, W., Johnson, D., \& BoyesBraEM, P. (1976). Basic objects in natural categories. Cognitive Psychology, 8, 382-439.

Smith, L. B. (1983). Development of classification: The use of similarity and dimensional relations. Journal of Experimental Child Psychology, 36, 150-178.

Treisman, A. (1986). Properties, parts, and objects. In K. R. Boff, L. Kaufman, \& P. Thomas (Eds.), Handbook of perception and performance (Vol. 2, pp. 35-1-35-70). New York: Wiley.

TVERSKY, B. (1989). Parts, partonomies, and taxonomies. Developmental Psychology, 25, 983-995.

TVERSKY, B., \& HEMENWAY, K. (1984). Objects, parts, and categories. Journal of Experimental Psychology: General, 113, 169-193.

VuRPILLOT, E. (1976). The visual world of the child. New York: International Universities Press.

W ARD, T. B. (1989). Analytic and holistic modes of processing in category learning. In B. E. Shepp \& S. Ballesteros (Eds.), Object perception: Structure \& process (pp. 387-419). Hillsdale, NJ: Erlbaum.

WERNER, H. (1957). Comparative psychology of mental development (rev. ed.). New York: International Universities Press.

(Manuscript received July 20, 1992.) 